\title{
Multiple Powers Allocation Strategy under Sensing/Transmission Frame Structure in Cognitive Radio Networks
}

\author{
Dao-Yuan Ren, Shou-Yi Yang, Fang Wang \\ School of Information Engineering, Zhengzhou \\ University, Zhengzhou 450001, China \\ E-mail:1192746122@qq.com
}

\author{
Liu-Jie Ji \\ Henan Radio \& Television University, \\ Zhengzhou 450001, China \\ E-mail:1192746122@qq.com
}

\begin{abstract}
In the traditional opportunity spectrum access cognitive radio system(OSA), the state of the primary user is assumed to be constant during the entire frame duration, the secondary user accesses licensed band with proper power only when primary user is detected idle. In fact, the state of the primary user may changes at any time, on the premise of ensure that the quality of service of primary user and secondary user to make full use of the channel, we proposed a new power allocation strategy based on the four states and three power sunder sensing/transmission frame structure. In this strategy, secondary user is assigned to three different types of access power for the four kinds of states in the process of sensing, considering the effect of activity of the primary user to the system throughput, adopting frame structure that maximize the sensing period and the data transmission period at the same time, to avoid the sensing-throughput tradeoff, aiming to achieve the maximize throughput. The simulation results show that compared to the new power allocation strategyin this paper and conventional power allocation strategy, proposed strategy in this paper has obvious improvement for throughput. In addition, the proposed system throughput is associated with the target detection probability and the primary user's signal- to-noise ratio received at the secondary user.
\end{abstract}

Keywords-cognitive radio; power allocation; spectrum sensing; spectrum sharing; throughput maximization

\section{INTRODUCTION}

With the rapid development of wireless communication technology, the requirements of information transmission speed and quality unceasing enhancement, the spectrum scarcity has been significantly amplified out.Cognitive radio technology ${ }^{[1]-[3]}$ is a effective method to improve spectrum utilization, allowing secondary users to access the licensed frequency band under the condition of protecting the quality of service (QOS) of the primary users, greatly improving the spectrum efficiency and system capacity. Now the main spectrum access technology are opportunity spectrum $\operatorname{access}(\mathrm{OSA})^{[4]-[5]}$ and spectrum sharing(SS) ${ }^{[6]-[8]}$.

At present, most of the research assume that the state of the primary user not changed in a frame, and to do two powers optimization for secondary user to access licensed band, namely that secondary user uses low power to access when primary user is detected busy; Otherwise using the high power. But the primary user stats may change in a frame, if secondary user uses a high power to access, May be causing a larger interference to primary user when primary user from busy to idle; If secondary user uses a low power to access, may be causing that secondary user failed to make full use of licensed band when primary from busy to idle. In [9], discussing the problem of sensing-throughput tradeoff on the promise of fixing high target detection probability, in order to determine the optimal time of sensing to maximize the system throughput. The new frame structure proposed in [10] (named sensing/transmission frame structure in this paper), this frame structure overcome the problem of sensing-throughout tradeoff, the simulation results show that this method compared with the conventional frame structure to increase the system throughput.The influence of activity of primary user to cognitive network is studied in [11]-[12],primary user can random arrive and departure during the frame with the actual environment. Among them, in [12] discussing the influence of multiple primary users of random arrive and departure to throughput. The conventional power allocation strategy is proposed in [13], which based on sensing/transmission frame structure with four states and two powers, achievable throughput have a sharply higher than[9] -[11], but the power allocation strategy in [13] still can be further optimized in regard to the power of secondary user access the licensed band.

In this paper, we proposed a new power allocation strategy which based on the sensing/transmission frame structure with four states and three powers in spectrum sharing cognitive radio networks, to do three powers optimization for secondary user to access the licensed band, and take into account the primary user activity, to maximize the system throughput as the optimization goal. The channel capacity and system achievable throughput of new power allocation strategy are derived in theory, in addition we discuss the influence of the target probability of detection and the primary user's signal-to-noise ratio received at the secondary user to achievable throughput, and compared to the conventional power allocation strategy in [13].

\section{SYSTEM MODEL AND DERIVATION}

We consider the cognitive radio system presented in Fig.1.Let $\mathrm{g}$ and $\mathrm{h}$ denotes the channel from the secondary transmitter (SU-TX) to the secondary receiver (SU-RX) and the primary receiver (PU-RX), respectively. The channels $g$ and $\mathrm{h}$ are assumed to be ergodic, stationary and known at the secondary users. Whereas the noise is assumed to be circularly symmetric complex Gaussian (CSCG) with mean zero and variance $\sigma_{n}^{2}$, namely $C N\left(0, \sigma_{n}^{2}\right)$. 


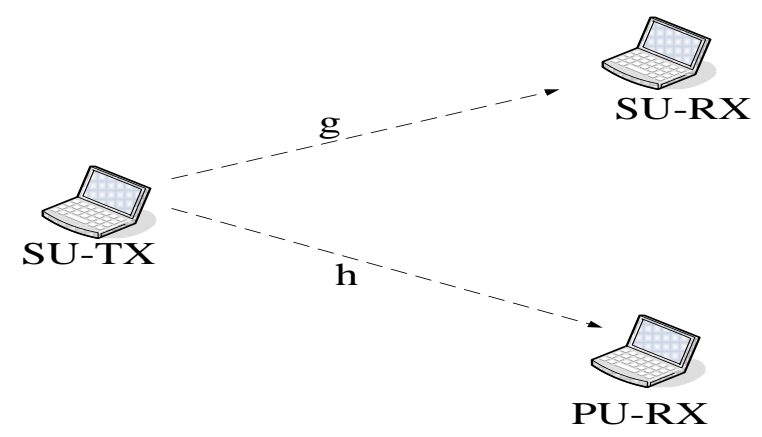

Figure 1. System model.

\section{A. Sensing/Transmission Frame Structure}

Sensing/transmission frame structure is adopted in this paper in Fig.2,achieving it rely on the special decoding device at secondary user receiver in Fig.3. Sensing/transmission frame structure exhibits several advantages, such as data transmission period and sensing period cover the whole duration of the frame, and effectively solved the problems of the tradeoff of sensing-transmission.

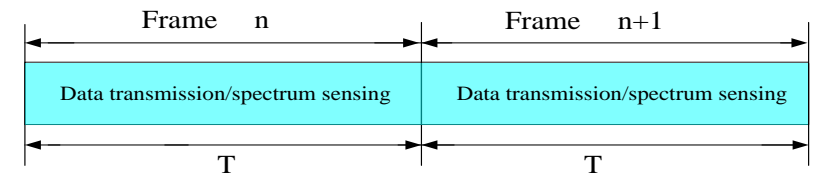

Figure 2. Sensing/Transmission Frame Structure.

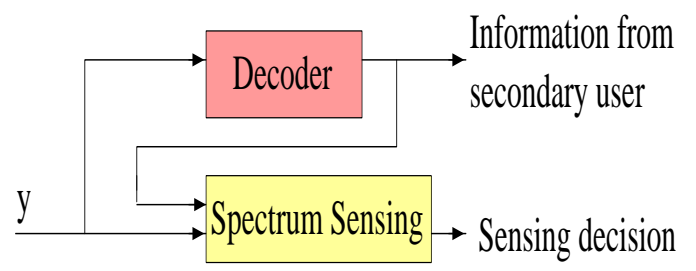

Figure 3. Decoder of secondary user receiver

The received signal at the secondary user is given by

$$
y=\theta x_{p}+x_{s}+n
$$

where $\theta$ denotes the actual status of the frequency band ( $\theta=1$ when it is active, $\theta=0$ when it is idle), $x p$ denotes the received signal from the primary users that use the frequency band, xs represents the signal from the secondary transmitter and finally $\mathrm{n}$ denotes the additive noise.

\section{B. The Four State Model Based on Sensing/Transmission Frame Structure}

In the sensing/transmission frame structure, the primary user traffic is modeled as a 1-0 random process, where ' 1 ', represents primary busy and ' 0 ' represents primary idle. Exponential holding time is assumed for each status, with mean parameter $\lambda$ for ' 1 ' and mean parameter $\mu$ for ' 0 ' [11]. At any time instant, the primary user is busy with probability $p_{b}=\frac{\lambda}{\lambda+\mu}$, and idle with probability $P_{e}=1-P_{b}$.The transition probability is given by [11]

$$
p(T)=\left(\begin{array}{ll}
p_{00}(T) & p_{01}(T) \\
p_{10}(T) & p_{11}(T)
\end{array}\right)=\frac{1}{\lambda+\mu}\left(\begin{array}{cc}
\lambda+\mu e^{-(\lambda+\mu) T} & \mu-\mu e^{-(\lambda+\mu) T} \\
\lambda-\lambda e^{-(\lambda+\mu) T} & \mu+\lambda e^{-(\lambda+\mu) T}
\end{array}\right) .
$$

It is further assumed that the primary user state transition occurs at most once within each frame. The case of two transitions in one frame is also examined by simulation but is not analyzed. Based on the above assumptions, the quaternary hypothesis testing problem[13]given by(3)

\section{The Four States and Three Power Allocation Strategy \\ Based on Sensing/Transmission Frame Structure}

In the conventional power allocation strategy,primary user is assumed that state remains unchanged in the frame,so when primary user is detected busy,secondary user use a low transmit power; otherwise,secondary user use a high transmit power.In fact,state of primary user may have changed in any time, if secondary user in a high power to access, May be causing a larger interference to primary user when primary user from busy to idle; If secondary user in a low power to access, may be causing that secondary user failed to make full use of licensed band when primary from busy to idle.Giving consideration to the above two cases,we proposed a new power allocation strategy.

$$
y= \begin{cases}\sum_{i=1}^{J} n_{i}^{2}, & H_{00}, \\ \sum_{i=1}^{d}\left(x_{p i}+n_{i}\right)^{2}+\sum_{i=d+1}^{J} n_{i}^{2}, & H_{10}, \\ \sum_{i=1}^{J}\left(x_{p i}+n_{i}\right)^{2}, & H_{11}, \\ \sum_{i=1}^{a} n_{i}^{2}+\sum_{i=a+1}^{J}\left(x_{p i}+n_{i}\right)^{2}, & H_{01} .\end{cases}
$$

In this paper, assuming that primary user state transition occurs at most once within adjacent two frame, and calculate throughput of the latter frame, secondary user access licensed band by the spectrum sharing model, as followH10state: The primary user is busy for $\mathrm{d}$ samples and then stay idle during the rest of the frame, in this case, secondary user use a high transmit power p0;H01 state: The primary user is idle for a samples and then stay busy during the rest of the frame, in this case, secondary user use a low transmit power $\mathrm{p} 1 ; \mathrm{H} 00$ state: the primary user is always idle during the former frame, it could remain idle state or from idle to busy, in this case, secondary user use a medium transmit power pm;H11 state: this state is similar to state $\mathrm{H} 00$, secondary user use a medium transmit power $\mathrm{pm}$. Among them: $\mathrm{p} 1<\mathrm{pm}<\mathrm{p} 0$.

The unconditional probability of false alarm and the unconditional probability of detection can be found in [13] as follow 


$$
\begin{gathered}
P_{f a N}(\varepsilon, J)=\frac{P_{H_{00}}(T) P_{f a H_{00}}(\varepsilon, J)}{P_{H_{00}}(T)+P_{H_{10}}(T)}+\frac{P_{H_{10}}(T) P_{f a H_{10}}(\varepsilon, J)}{P_{H_{00}}(T)+P_{H_{10}}(T)}, \\
P_{d N}(\varepsilon, J)=\frac{P_{H_{11}}(T) P_{d H_{11}}(\varepsilon, J)}{P_{H_{11}}(T)+P_{H_{01}}(T)}+\frac{P_{H_{01}}(T) P_{d H_{01}}(\varepsilon, J)}{P_{H_{11}}(T)+P_{H_{01}}(T)},
\end{gathered}
$$

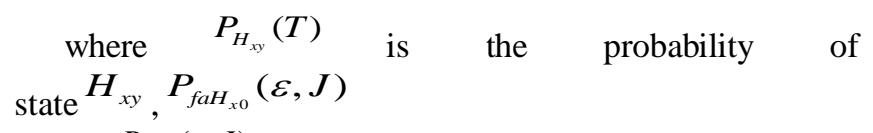

and $P_{d H_{0 y}}(\varepsilon, J)$ respectively is the conditional probability of state $H_{x 0}$ and $H_{0 y}, x, y \in\{0,1\}$.

Once the spectrum sensing in (3) is completed, the secondary transmission stats according to the sensing information to decide transmit power, as followH00 state: The channel capacity is affected by the primary user traffic, and the instantaneous channel capacity of primary user remain idle in a later frame system can be derived as

$$
K_{H_{00}}=\log _{2}\left(1+\frac{g_{s s} p_{m}}{N_{\mathrm{o}}}\right)
$$

The instantaneous channel capacity of primary user from idle to busy in a later frame system can be derived as

$$
K_{H_{00}}(a)^{\prime}=\log _{2}\left(1+\frac{g_{s s} p_{m}}{N_{0}+\frac{J-a}{J} g_{p s} p_{u}, \quad 0 \leq a \leq J,}\right.
$$

N0 is additive white Gaussian noise (AWGN), gss is channel gain of channel g, gps is the channel gain of primary user to secondary user. Therefore, the achievable throughput can be derived as

$$
\begin{aligned}
R_{H_{00}} & =P_{H_{00}}^{2}(T) K_{H_{00}}+P_{H_{00}}(T) P_{H_{01}}(T) K_{H_{00}}(a)^{\prime} \\
& =\alpha_{1} K_{H_{00}}+\alpha_{2} K_{H_{00}}(a)^{\prime}
\end{aligned}
$$

Where $\alpha_{1}=P_{H_{00}}{ }^{2}(T), \alpha_{2}=P_{H_{00}}(T) P_{H_{01}}(T), \alpha_{1}$ and $\alpha_{2}$ respe cti-vely corresponding to the probability of channel capacity $K_{H_{00}}$ and $^{K_{H_{00}}(a)^{\prime}}$. H01 state: according to the correctness of detection of the primary user by secondary user, we divided it into two case. The instantaneous channel capacity of wrong detect by secondary user can be derived as

$$
K_{H_{01}}=\log _{2}\left(1+\frac{g_{s s} p_{0}}{N_{0}+g_{p s} p_{u}}\right)
$$

The instantaneous channel capacity of right detect by secondary user can be derived as

$$
K_{H_{01}}^{\prime}=\log _{2}\left(1+\frac{g_{s s} p_{1}}{N_{0}+g_{p s} p_{u}}\right)
$$
follow

$$
\begin{aligned}
R_{H_{01}} & =P_{H_{01}}(T)\left[1-P_{d N}(\varepsilon, J)\right] K_{H_{01}}+P_{H_{01}}(T) P_{d N}(\varepsilon, J) K_{H_{01}}{ }^{\prime} \\
& =\alpha_{3} K_{H_{01}}+\alpha_{4} K_{H_{01}}{ }^{\prime},
\end{aligned}
$$

Where $\alpha_{3}=P_{H_{01}}(T)\left[1-P_{d N}(\varepsilon, J)\right], \alpha_{4}=P_{H_{01}}(T) P_{d N}(\varepsilon, J), \alpha_{3}$ and $\alpha_{4}$ respectively corresponding to the probability of channel capacity $K_{H_{01} \text { and }} K_{H_{01}}{ }^{\prime}$.H11 state: similar to state H00, the channel capacity is affected by the primary user traffic, and the instantaneous channel capacity of primary user remain busy in a later frame system can be derived as

$$
K_{H_{11}}=\log _{2}\left(1+\frac{g_{s s} p_{m}}{N_{0}+g_{p s} p_{u}}\right)
$$

The instantaneous channel capacity of primary user from busy to idle in a later frame system can be derived as

$$
K_{H_{11}}(d)^{\prime}=\log _{2}\left(1+\frac{g_{s s} p_{m}}{N_{0}+\frac{d}{J} g_{p s} p_{u}}\right), \quad 0 \leq d \leq J,
$$

Therefore the achievable throughput can be derived as

$$
\begin{aligned}
R_{H_{11}} & =P_{H_{11}}^{2}(T) K_{H_{11}}+P_{H_{11}}(T) P_{H_{10}}(T) K_{H_{11}}(d)^{\prime} \\
& =\beta_{1} K_{H_{11}}+\beta_{2} K_{H_{11}}(d)^{\prime}
\end{aligned}
$$

Where $\beta_{1}=P_{H_{11}}{ }^{2}(T), \beta_{2}=P_{H_{11}}(T) P_{H_{10}}(T), \beta_{1}$ and $\beta_{2}$ respective ly corresponding to the probability of channel capacity $K_{H_{11}}$ and $K_{H_{11}}(d)^{\prime}$.H10 state: according to whether occurring false alarm for the primary user by secondary user, we divided it into two case. The instantaneous channel capacity of no false alarm occurring can be derived as

$$
K_{H_{10}}=\log _{2}\left(1+\frac{g_{s s} p_{0}}{N_{0}}\right)
$$

The instantaneous channel capacity of false alarm occurring can be derived as

$$
K_{H_{10}}{ }^{\prime}=\log _{2}\left(1+\frac{g_{s s} p_{1}}{N_{0}}\right)
$$

Therefore the achievable throughput can be derived as

$$
\begin{aligned}
R_{H_{10}} & =P_{H_{10}}(T)\left[1-P_{f a N}(\varepsilon, J)\right] K_{H_{10}}+P_{H_{10}}(T) P_{f a N}(\varepsilon, J) K_{H_{10}}{ }^{\prime} \\
& =\beta_{3} K_{H_{10}}+\beta_{4} K_{H_{10}}{ }^{\prime},
\end{aligned}
$$

Where $\beta_{3}=P_{H_{10}}(T)\left[1-P_{f a N}(\varepsilon, J)\right], \beta_{4}=P_{H_{10}}(T) P_{f a N}(\varepsilon, J), \beta_{3}$ and $\beta_{4}$ respectively corresponding to the probability of channel 
capacity $K_{H_{10}}$ and $K_{H_{10}}{ }^{\prime}$. Being able to get achievable throughput of cognitive system using with (5)-(16), as follow

$$
R_{N}=R_{H_{00}}+R_{H_{01}}+R_{H_{11}}+R_{H_{10}}
$$

(17) can be rewritten as:

$$
\begin{aligned}
& \operatorname{maximize}_{\{P\}} R_{N}=E_{g_{s}, \gamma_{p . p}}\left\{\alpha_{1} K_{H_{\infty}}+\alpha_{2} K_{H_{\infty 0}}(a)^{\prime}+\alpha_{3} K_{H_{01}}+\alpha_{4} K_{H_{01}}{ }^{\prime}\right. \\
& \left.+\beta_{1} K_{H_{11}}+\beta_{2} K_{H_{11}}(d)^{\prime}+\beta_{3} K_{H_{10}}+\beta_{4} K_{H_{10}}{ }^{\prime}\right\},
\end{aligned}
$$

Subject to

$$
\begin{aligned}
& E_{g_{s s}, g_{p p}}\left\{\left(\alpha_{1}+\alpha_{2}+\beta_{1}+\beta_{2}\right) p_{m}+\left(\alpha_{3}+\beta_{3}\right) p_{0}+\left(\alpha_{4}+\beta_{4}\right) p_{1}\right\} \leq p_{a v}, \\
& E_{g_{s s s}, g_{s p}}\left\{\left(\alpha_{2}+\beta_{1}+\beta_{2}\right) p_{m} g_{s p}+\alpha_{3} p_{0} g_{s p}+\alpha_{4} p_{1} g_{s p}\right\} \leq \Gamma,
\end{aligned}
$$

(19) and (20) respectively represents average transmit and interference power constraint, $\mathrm{p} 0 \geq 0, \mathrm{pl} \geq 0, \mathrm{pm} \geq 0$, gsp is channel gain of channel $h$.

The Lagrangian with respect to the transmit powersp0, $\mathrm{p} 1$ and $\mathrm{pm}$ is given by

$$
\begin{aligned}
& L\left(p_{0}, p_{1}, p_{m}, \lambda, \mu\right) \\
& =E_{g_{s}, g_{0, p}}\left\{\alpha_{1} K_{H_{00}}+\alpha_{2} K_{H_{00}}(a)^{\prime}+\alpha_{3} K_{H_{01}}+\alpha_{4} K_{H_{01}}{ }^{\prime}\right. \\
& \left.+\beta_{1} K_{H_{11}}+\beta_{2} K_{H_{11}}(d)^{\prime}+\beta_{3} K_{H_{10}}+\beta_{4} K_{H_{10}}^{\prime}\right\} \\
& -\lambda\left[E_{g_{s, s}, g_{m}}\left\{\left(\alpha_{1}+\alpha_{2}+\beta_{1}+\beta_{2}\right) p_{m}+\left(\alpha_{3}+\beta_{3}\right) p_{0}+\left(\alpha_{4}+\beta_{4}\right) p_{1}\right\}-p_{a v}\right] \\
& -\mu\left[E_{s_{s s}, g_{p q}}\left\{\left(\alpha_{2}+\beta_{1}+\beta_{2}\right) p_{m} g_{s p}+\alpha_{3} p_{0} g_{s p}+\alpha_{4} p_{1} g_{s p}\right\}-\Gamma\right],
\end{aligned}
$$

Whereas the dual function can be obtained by

$$
d(\lambda, \mu)=\sup _{p_{0}, p_{1}, p_{m}} L\left(p_{0}, p_{1}, p_{m}, \lambda, \mu\right)
$$

In order to calculate the dual function $\mathrm{d}(\lambda, \mu)$, the supremum of the Lagrangian with respect to the transmit powers $\mathrm{p} 0, \mathrm{p} 1$ and $\mathrm{pm}$ needs to be obtained. We therefore apply the primal-dual-decomposition method[14], which facilitates the solution of the joint optimization problem by decomposing it into three convex single-variable optimization problems, one for each of the transmit powersp0, p1 and pm, as follows:

\section{Subproblem 1:}

$$
\begin{aligned}
& \underset{\left\{p_{0} \geq 0\right\}}{\max i \min } e \\
& f_{1}\left(p_{0}\right)= \\
& \quad E_{g_{s s}, g_{s p}}\left\{\alpha_{3} \log _{2}\left(1+\frac{g_{s s} p_{0}}{N_{0}+g_{p s} p_{u}}\right)+\beta_{3} \log _{2}\left(1+\frac{g_{s s} p_{0}}{N_{0}}\right)\right\} \\
& \quad-\lambda E_{g_{s s}, g_{s p}}\left\{\left(\alpha_{3}+\beta_{3}\right) p_{0}\right\}-\mu E_{g_{s s}, g_{s p}}\left\{\alpha_{3} p_{0} g_{s p}\right\} .
\end{aligned}
$$

\section{Subproblem 2:}

$$
\begin{aligned}
\underset{\left\{p_{1} \geq 0\right\}}{\max i \min } & e \\
f_{2}\left(p_{1}\right)= & E_{g_{s s}, g_{s p}}\left\{\alpha_{4} \log _{2}\left(1+\frac{g_{s s} p_{1}}{N_{0}+g_{p s} p_{u}}\right)+\beta_{4} \log _{2}\left(1+\frac{g_{s s} p_{1}}{N_{0}}\right)\right\} \\
& -\lambda E_{g_{s s}, g_{s p}}\left\{\left(\alpha_{4}+\beta_{4}\right) p_{1}\right\}-\mu E_{g_{s s}, g_{s p}}\left\{\alpha_{4} p_{1} g_{s p}\right\} .
\end{aligned}
$$

\section{Subproblem 3:}

$$
\begin{aligned}
f_{3}\left(p_{m}\right)=E_{g_{s s}, g_{s p}} & \left\{\alpha_{1} \log _{2} \geq \log _{2}\left(1+\frac{g_{s s} p_{m}}{N_{0}}\right)+\alpha_{2} \log _{2}\left(1+\frac{g_{s s} p_{m}}{N_{0}+\frac{J-a}{J} g_{p s} p_{u}}\right)\right. \\
& \left.+\beta_{1} \log _{2}\left(1+\frac{g_{s s} p_{m}}{N_{0}+g_{p s} p_{u}}\right)+\beta_{2} \log _{2}\left(1+\frac{g_{s s} p_{m}}{N_{0}+\frac{d}{J} g_{p s} p_{u}}\right)\right\} \\
& -\lambda E_{g_{s s} g_{p p}}\left\{\left(\alpha_{1}+\alpha_{2}+\beta_{1}+\beta_{2}\right) p_{m}\right\}-\mu E_{g_{s s}, g_{p}}\left\{\left(\alpha_{2}+\beta_{1}+\beta_{2}\right) p_{1} g_{s p}\right\} .
\end{aligned}
$$

After forming their Lagrangian functions and applying the Karush-Kuhn-Tucker (KKT) conditions, the optimal powersp $0, \mathrm{p} 1$ and $\mathrm{pm}$ for given $\lambda, \mu$ are given by

$$
\begin{gathered}
p_{\mathrm{o}}=\left[\frac{A_{\mathrm{o}}+\sqrt{\Delta_{\mathrm{o}}}}{2}\right]^{+}, p_{1}=\left[\frac{A_{1}+\sqrt{\Delta_{1}}}{2}\right]^{+}, \\
p_{m}=-\frac{B_{0}}{4}+\frac{1}{2} \sqrt{\frac{B_{0}^{2}}{4}-\frac{2 B_{1}}{3}+\Delta_{4}}+\frac{1}{2} \sqrt{\frac{B_{0}^{2}}{2}-\frac{4 B_{1}}{3}-\Delta_{4}+\frac{-B_{0}^{3}+4 B_{0} B_{1}-8 B_{2}}{\sqrt{\frac{B_{0}^{2}}{4}-\frac{2 B_{1}}{3}+\Delta_{4}}}}
\end{gathered}
$$

Where ${ }^{[x]^{+}}=\max (0, x)$. The parameters in the formula (26) can be seen at appendix.

In order to determine the optimal power allocation strategy, the optimal values of the Lagrangian multipliers $\lambda$, $\mu$ that minimize the dual function $\mathrm{d}(\lambda, \mu)$ need to be found. The ellipsoid method[15] is used here to find the optimal solution, which requires the subgradient of the dual function $\mathrm{d}(\lambda, \mu)$. The latter is given by the following proposition.

Proposition 1: The subgradient of the dual function $\mathrm{d}(\lambda, \mu)$ is $[D, E]$, where Dand Eis given by

$$
\begin{gathered}
D=p_{a v}-E_{g_{s s}, g_{s p}}\left\{\left(\alpha_{1}+\alpha_{2}+\beta_{1}+\beta_{2}\right) p_{m}+\left(\alpha_{3}+\beta_{3}\right) p_{0}+\left(\alpha_{4}+\beta_{4}\right) p_{1}\right\}, \\
E=\Gamma-E_{g_{s s}, g_{s p}}\left\{\left(\alpha_{2}+\beta_{1}+\beta_{2}\right) p_{m} g_{s p}+\alpha_{3} p_{0} g_{s p}+\alpha_{4} p_{1} g_{s p}\right\} .
\end{gathered}
$$

Where $\lambda \geq 0$ and $\mu \geq 0, p 0, p 1$ and pm denote the optimal power allocation in (22) for fixed $\lambda$ and $\mu$.

The algorithm that acquires the optimal power allocation strategy that maximizes the ergodic capacity of the proposed spectrum sharing cognitive radio system is presented in the following table. 
TABLE I. ALGORITHM

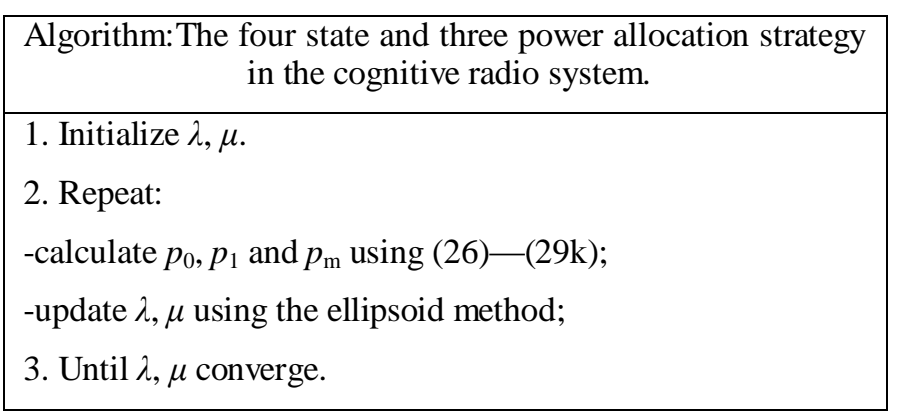

\section{NUMERICAL RESULTS AND DISCUSSION}

In this section, we present simulation results for the new power allocation strategy and compare it with the conventi-onal power allocation strategy under sensing/transmission frame structure. The frame duration is set to $\mathrm{T}=100 \mathrm{~ms}$, the sampling frequency fs $=6 \mathrm{MHZ}$, gss, gps and gspare exponentially distributed, and $\mathrm{E}\{$ gss $\}=\mathrm{E}\{\mathrm{gsp}\}=$ $\mathrm{E}\{\mathrm{gps}\}=1$.

In Fig.4, we present the achievable throughput versus the additional channel power gain gsp of SU-TX to PU-TX for the new power allocation strategy and the conventional power allocation strategy in [13]. The target probability of detection is set to $\mathrm{Pd}=0.99$, the primary user's signal-tonoise ratio (SNR) received at the secondary users is set to $\gamma \mathrm{p}=-10 \mathrm{~dB}$, and the mean channel holding time $\lambda$ and $\mu$ is set to $\lambda=\mu=10$. One can clear see that the achievable throughput of the new power allocation strategy is higher compared to the conventional power allocation strategy. This is due to on the promise of protecting the quality of service of primary users, the new power allocation strategy allows secondary user access licensed band for sending data when primary user is busy, so improving the system achievable throughput. Moreover, it can be seen from Fig.5 that with the increase of gsp, the system achievable throughput decrease, which is due to with increasing the channel gain gsp, the interference of cognitive users to primary users also increase, but the primary users can withstand interference is limited, in order to protect the performance of the primary user, appropriate to reduce the sending power of cognitive users, thus caused the fall of system achievable throughput.

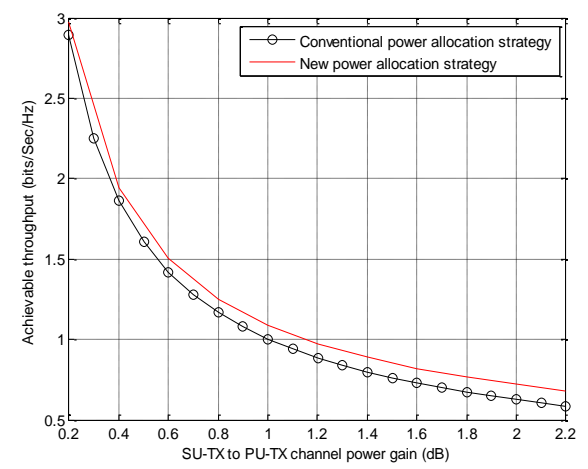

Figure 4. Achievable throughput of the new power allocation strategy and the conventional power allocation strategy versus the additional channel power gain.
In Fig.5, the achievable throughput versus the target detection probability $\mathrm{Pd}$ for the new power allocation strategy and the conventional power allocation strategy. $\gamma \mathrm{p}$ is set to $\gamma \mathrm{p}=-10 \mathrm{~dB}$ and the mean channel holding time $\lambda$ and $\mu$ is set to $\lambda=\mu=10$. One can clear see that with the improvement of target detection probability, whether it is a new power allocation strategy or a traditional power allocation strategy, the system achievable throughput will present a downward trend. Moreover the system of new power allocation strategy can achieve throughput significantly higher than under the same conditions of the system of traditional power allocation strategy. This is due to under the high probability of target detection, the new power allocation strategy based on three power allocation not only can better protect the user, also can obtain higher throughput.

In Fig.6, we present the achievable throughput versus the additional channel power gain gsp of SU-TX to PU-TX for the new power allocation strategy and the conventional power allocation strategy for the different $\gamma$ p. The target probability of detection is set to $\mathrm{Pd}=0.99$ and the mean channel holding time $\lambda$ and $\mu$ is set to $\lambda=\mu=10$. We can clear see that with the reduce of the $\gamma$ p, the system achievable throughput is also have obvious drop. This is due to with the reduce of primary user's SNR received at the secondary user, the effect of sensing of secondary user will fall, this will lead to false-alarm probability of cognitive users increase, therefore the secondary user is difficult to send the data access licensed channel, so cause the loss of system achievable throughput. Moreover the system of new power allocation strategy can achieve throughput higher than under the same conditions of the system of traditional power allocation strategy.

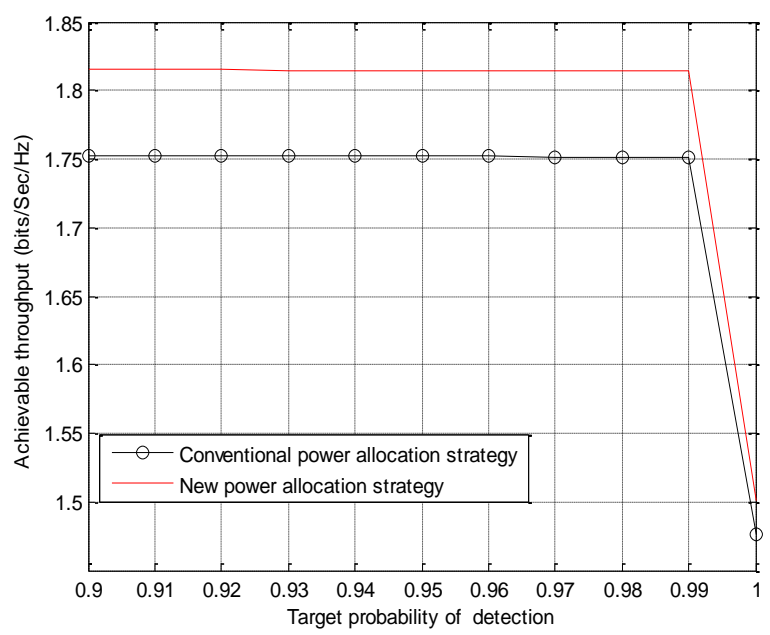

Figure 5. Achievable throughput of the new power allocation strategy and the conventional power allocation strategy versus the target probability of detection. 


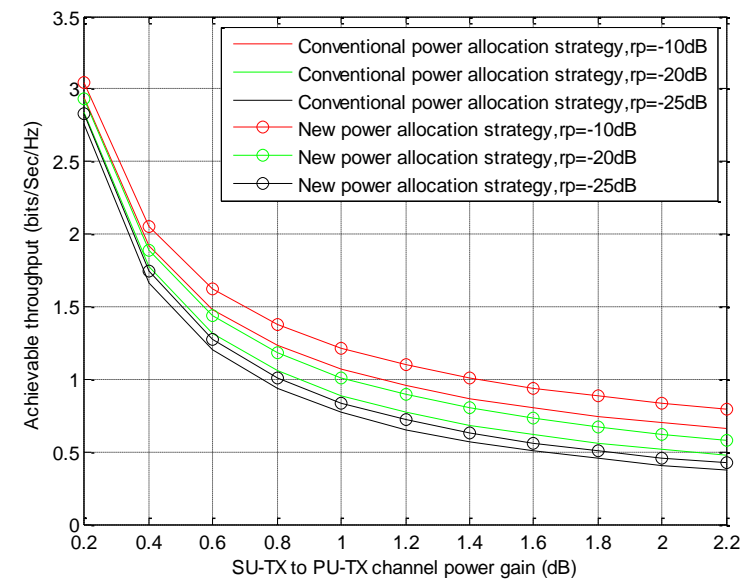

Figure 6. Achievable throughput of the new power allocation strategy and the conventional power allocation strategy versus the additional channel power gain gsp for the differentyp.

\section{CONCLUSIONS}

In this paper, we proposed a new power allocation strategy in the spectrum sharing access model, in a more realistic scenario, consideringthat the primary user arrives or leaves at any time, we distribute three different types of access power for four kinds of states of sensing, aiming to maximize the systemthroughput. Simulation results show thatcompared with the conventional power allocation strategy, the new power allocation strategy has the obvious enhancement and improvement in achievable throughput. Moreover the target probability of detection and the primary user's signal-to-noise ratio received at the secondary user have an impact on achievable throughput, but the overall performance of the new power allocation strategy is still superior to the conventional power allocation strategy in[13].

\section{APPENDIX}

The parameters in the formula (26) as shown:

$$
\begin{gathered}
A_{0}=\frac{\alpha_{3}+\beta_{3}}{\left[\lambda\left(\alpha_{3}+\beta_{3}\right)+\mu \alpha_{3} g_{s p}\right] \ln 2}-\frac{2 N_{0}+g_{p s} p_{u}}{g_{s s}}, \\
\Delta_{0}=A_{0}-\frac{g_{s s}}{4}\left\{\frac{N_{0}\left(N_{0}+g_{p s} p_{u}\right)}{g_{s s}}-\frac{\alpha_{3} N_{0}+\beta_{3}\left(N_{0}+g_{p s} p_{u}\right)}{\left[\lambda\left(\alpha_{3}+\beta_{3}\right)+\mu \alpha_{3} g_{s p}\right] \ln 2}\right\}, \\
A_{1}=\frac{\alpha_{4}+\beta_{4}}{\left[\lambda\left(\alpha_{4}+\beta_{4}\right)+\mu \alpha_{4} g_{s p}\right] \ln 2}-\frac{2 N_{0}+g_{p s} p_{u}}{g_{s s}}, \\
\Delta_{1}=A_{1}-\frac{g_{s s}}{4}\left\{\frac{N_{0}\left(N_{0}+g_{p s} p_{u}\right)}{g_{s s}}-\frac{\alpha_{4} N_{0}+\beta_{4}\left(N_{0}+g_{p s} p_{u}\right)}{\left[\lambda\left(\alpha_{4}+\beta_{4}\right)+\mu \alpha_{4} g_{s p}\right] \ln 2}\right\}, \\
\Delta_{4}=\frac{\sqrt[3]{2} \Delta_{2}}{3 \sqrt[3]{\Delta_{3}+\sqrt{-4 \Delta_{2}^{3}+\Delta_{3}^{2}}}}+\frac{\sqrt[3]{\Delta_{3}+\sqrt{-4 \Delta_{2}^{3}+\Delta_{3}^{2}}}}{3 \sqrt[3]{2}}, \\
\Delta_{2}=B_{1}^{2}-3 B_{0} B_{2}+12 B_{3},
\end{gathered}
$$

$$
\begin{gathered}
\Delta_{3}=2 B_{1}^{3}-9 B_{0} B_{1} B_{2}+27 B_{2}^{2}+27 B_{0}^{2} B_{3}-72 B_{1} B_{3}, \\
B_{0}=a^{\prime}+b^{\prime}+c^{\prime}+d^{\prime}-\frac{\alpha_{1}+\alpha_{2}+\beta_{1}+\beta_{2}}{M \ln 2}, \\
B_{1}=a^{\prime} b^{\prime}+a^{\prime} c^{\prime}+a^{\prime} d^{\prime}+b^{\prime} c^{\prime}+b^{\prime} d^{\prime}+c^{\prime} d^{\prime}-\frac{1}{M \ln 2}\left[\alpha_{1}\left(b^{\prime}+c^{\prime}+d^{\prime}\right)\right. \\
\left.+\alpha_{2}\left(a^{\prime}+c^{\prime}+d^{\prime}\right)+\beta_{1}\left(a^{\prime}+b^{\prime}+d^{\prime}\right)+\beta_{2}\left(a^{\prime}+b^{\prime}+c^{\prime}\right)\right], \\
B_{2}=a^{\prime} b^{\prime} c^{\prime}+a^{\prime} b^{\prime} d^{\prime}+a^{\prime} c^{\prime} d^{\prime}+b^{\prime} c^{\prime} d^{\prime}-\frac{1}{M \ln 2}\left[\alpha_{1}\left(b^{\prime} c^{\prime}+b^{\prime} d^{\prime}+c^{\prime} d^{\prime}\right)\right. \\
\left.+\alpha_{2}\left(a^{\prime} c^{\prime}+a^{\prime} d^{\prime}+c^{\prime} d^{\prime}\right)+\beta_{1}\left(a^{\prime} b^{\prime}+a^{\prime} d^{\prime}+b^{\prime} d^{\prime}\right)+\beta_{2}\left(a^{\prime} b^{\prime}+a^{\prime} c^{\prime}+b^{\prime} c^{\prime}\right)\right], \\
B_{3}=a^{\prime} b^{\prime} c^{\prime} d^{\prime}-\frac{1}{M \ln 2}\left(\alpha_{1} b^{\prime} c^{\prime} d^{\prime}+\alpha_{2} a^{\prime} c^{\prime} d^{\prime}+\beta_{1} a^{\prime} b^{\prime} d^{\prime}+\beta_{2} a^{\prime} b^{\prime} c^{\prime}\right),
\end{gathered}
$$

where $M=\lambda\left(\alpha_{1}+\alpha_{2}+\beta_{1}+\beta_{2}\right)+\mu\left(\alpha_{2}+\beta_{1}+\beta_{2}\right) g_{s p}$,

$$
\begin{aligned}
& \boldsymbol{a}^{\prime}=\frac{N_{\mathrm{O}}}{g_{s s}}, \quad b^{\prime}=\frac{N_{0}+\frac{J-a}{J} g_{p s} p_{u}}{g_{s s}}, \quad c^{\prime}=\frac{N_{0}+g_{p s} p_{u}}{g_{s s}}, \\
& d^{\prime}=\frac{N_{0}+\frac{d}{J} g_{p s} p_{u}}{g_{s s}} .
\end{aligned}
$$

\section{REFERENCE}

[1] Fed. Commun Comm., "Spectrum Policy Task Force Report,"Washington, DC, 02-155, Nov. 2002.

[2] J. Mitola,and G.Q.Maguire,Jr.,"Cognitive radios: Making software radio more personal,"IEEEPers. Commun., vol. 6, no. 4, pp. 13-18, Aug. 1999.

[3] S. Haykin," Cognitive radio: Brain-empowered wireless communications,” IEEE J. Sel. Areas Commun., vol. 23, no. 2, pp. 201-220, Feb. 2005.

[4] Q. Zhao and A. Swami,"A decision-theoretic framework for opportunistic spectrum access,"IEEE Trans. Wireless Commun. Mag.,vol. 14, no. 4, pp. 14-20, Aug. 2007.

[5] I. F. Akyildiz,W. Y. Lee,“ Next generationdynamic spectrum accesscognitive radio wireless networks,"Comput, Netw. 2006, 50 (13) :

2127-2159.

[6] L. Musavian and S. Aissa,"Ergodic and outage capacities of spectrum-sharing systems in fading channels," in Proc. IEEE GLOBECOM, Washington, DC, Nov. 2007, pp. 3327-3331.

[7] A. Ghasemi and E. S. Sousa, "Fundamental limits of spectrum-shar-ing in fading environments,"IEEE Trans. Wireless Commun., vol. 6, no. 2, pp. 649-658,Feb. 2007.

[8] M. Gastpar," On capacity under receive and spatial spectrum-sharing constraints,"IEEE Trans. Inf. Theory, vol.53, no. 2, pp. 471-487,Feb. 2007

[9] Y.-C. Liang, Y. Zeng, E. C. Y. Peh, and A. T. Hoang,“ Sensing-throu -ghput tradeoff for cognitive radio networks,"IEEE Trans. Wireless Commun., vol. 7, no. 4, pp. 1326-1337, Apr. 2008.

[10] S. Stotas, A. Nallanathan, "Overcoming the sensing-throughput tradeoff in cognitive radio networks,"IEEE International Conf Commun, Cape Town, South Africa: IEEE Press,2010:1-5.

[11] T. Liang, C. Yunfei,“ Effect of primary user traffic on sensing-throughput tradeoff for cognitive radios,"IEEE Trans. Wireless Commun, vol. 10, no. 4, Apr. 2011.

[12] H. Pradhan, S. S. Kalamkar," Sensing-Throughput Tradeoff in Cognitive Radio With Random Arrivals and Departures of Multiple Primary Users, "IEEE Commun Letters, vol.19, no.3,Mar. 2015. 
[13] F. Yanan, Y. Shouyi, “Effect of primary user traffic on throughput for novel cognitive radios,"ISSN,2013, 37 (23).

[14] D. P. Palomar and M. Chiang, "A tutorial on decomposition methods for network utility maximization,"IEEE J. Sel. Areas Commun.,vol.24, no.8, pp.1439-1451.Aug.2006.
A. Ben-Tal and A. Nemirovski, Lectures on Modern Convex Optimization: Analysis, Algorithms, and Engineering Applications. Philadelphia, PA: SIM,2001. 